\title{
Method of multiservice infrastructure decomposition with network resource slicing for IoT
}

\author{
Vasyl Romanchuk ${ }^{1, a}$, Mykola Beshley ${ }^{1, * b}$, Andriy Prislupskiy ${ }^{1, c}$, Halyna Beshley ${ }^{1, d}$ and \\ Oleksiy Panchenko, \\ ${ }^{1}$ Department of Telecommunications, Lviv Polytechnic National University, Lviv, Ukraine \\ a.romanchuk@lpnu.ua,b.beshlebmi@gmail.com, \\ c.pr.andrii@gmail.com,d.halink@ukr.net,e.oleksij@gmail.com \\ *corresponding author
}

Keywords: QoS parameters, virtualization, IoT slice, network resources, multiservice network.

Abstract: A new approach to the construction of a multiservice infrastructure with virtualization of network devices is proposed. Multiservice network with a virtualization is a network in which on one or several devices the virtualization mode is used. Virtualization of a network device involves the creation of two or more virtual network machines that serve as a switch or router with individual streaming services. The method of multiservice infrastructure decomposition of a network device with virtualization these resources is developed. This method ensure efficient allocation between different network streams and will allow to improve the quality of service of real-time streams with the provision of guaranteed QoS level of services that are sensitive to losses and delays. The additional novelty of our proposed approach is the additional IoT (Internet of Things) slice overlaying the traditional multiservice network.

Original article, Published date: 2018-06-19

DOI: 10.23977/iotea.2017.31003

ISSN: ISSN 2371-8609 (Print), ISSN 2371-8617 (Online)

https://www.clausiuspress.com/journal/IoTEA.html

\section{Introduction}

Modern telecommunication networks are aimed at providing a wide range of services to subscribers, which are characterized by various types of requirements for network resources [1]. The steady growth of information flows leads to the modernization of telecommunication networks both in terms of equipment of the network nodes and in relation to the continuous expansion of 
transmission channels throughput for an increasing amount of stream traffic and ensuring the quality of its transmission. Increasing the volume of real-time traffic (voice, video) and Internet of Things services (IoT services) creates a number of problems and requires the application of new methods for optimizing resources, management protocols and, consequently, replacing hardware [2-8]. The Internet of Things is a network of physical objects that can communicate and interact with the modern enterprise. It allows your businesses to learn key information about infrastructure, valuable assets, manufactured products, and more. With these new streams of data, you can unlock actionable intelligence to drive revenue growth, cut costs, and modernize how your business is done [9-10]. One of the methods for ensuring the quality of service of various types of traffic is the use of a system with priorities. At the same time, the system of service on the basis of fixed priorities has a significant disadvantage. This system is not able to provide the required QoS level for low priority flows [11-13]. Detailed analysis of these problems, optimization and configuration of used telecommunication equipment can provide growing requirements. At the same time, the cost of conducting such works is significantly lower than possible costs for the purchase and installation of new telecommunication equipment. The most promising solution for providing QoS and the balanced use of network resources, as well as the coherence between routing, switching, access control and resource reservation solutions is the use of network equipment virtualization technology IP network equipment optimization that prepares the basis for radical changes in the area of provision multimedia services through the Internet [14-17]. This new technology lets operators slice one physical network into multiple, virtual, end-to-end (E2E) networks, each logically isolated including device, access, transport and core network and dedicated for different types of services with different characteristics and requirements. For each network slice, dedicated resources (like resources within virtualized servers, network BW, QoS, etc.) are guaranteed [18]. As slices are isolated from each other, an error or fault occurred in one slice does not cause any effect on communication in other slices. Virtual switches open the way for new Internet services, which will be isolated from the traffic of other network services and will provide control over productivity, address administration, management tools and network security. In this regard the topic that is devoted to the research of virtualization of a network device in order to improve the quality of service of traffic in multiservice networks is actuality.

\section{Methods and Techniques}

To improve time rates of service quality and flexibility of managing network resources by developing a method decomposing the structure of a network device with virtualization of resources. The paper proposes a structural and functional model of a network node with static and dynamic virtualization of computing resources, which allows describing the process of adaptive virtualization of routers by optimizing the specified level of parameters for some types of service with a set that is inherent to them to ensure the quality of service and increase the overall stability of the system for cyberattacks. 


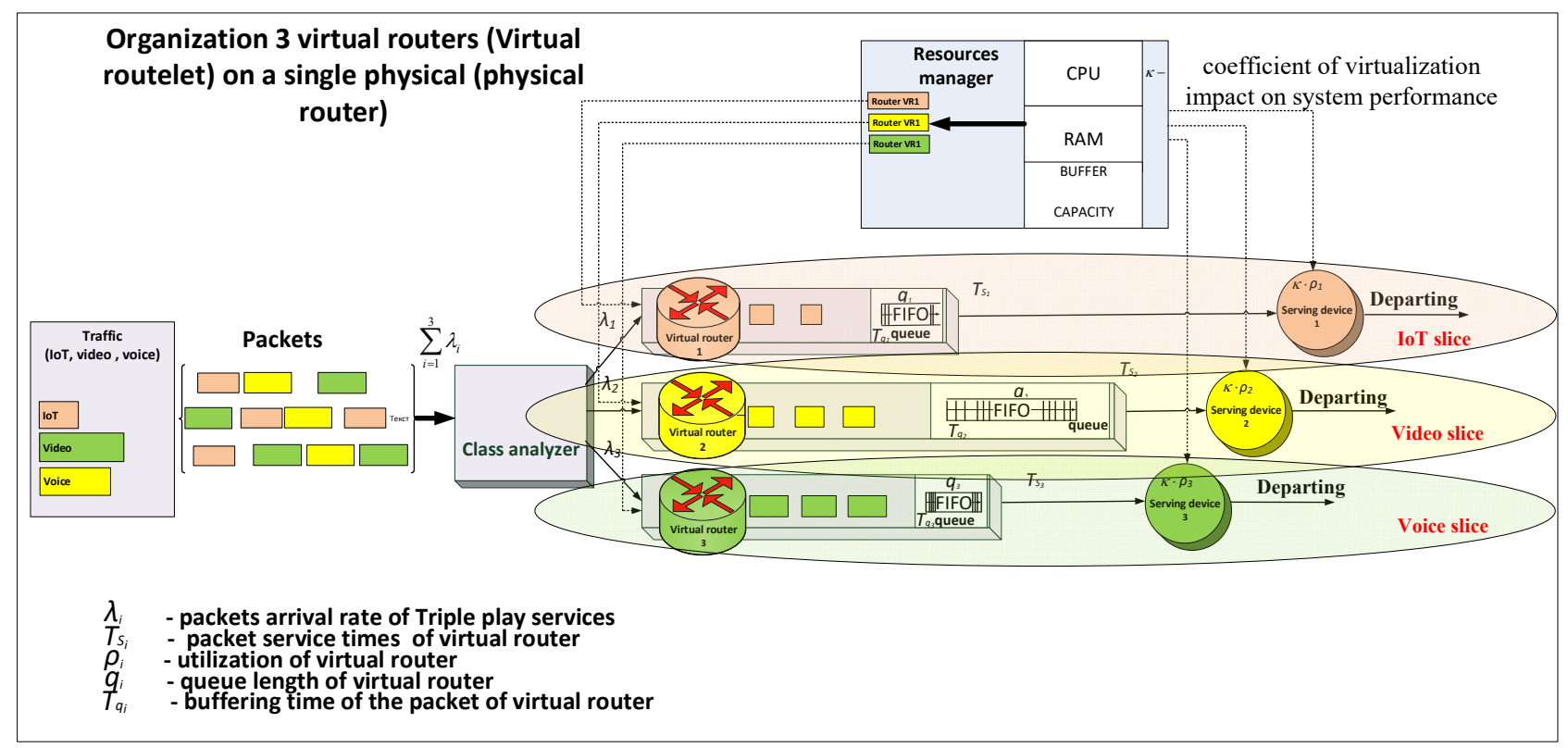

Figure 1: Structural and functional model of the network node with static and dynamic virtualization of computing resources

Virtualization of a network device involves the creation of two or more virtual hardware analogue routers. Each of these is intended for the individual maintenance of flows of the same type with the required level of QoS according to the formulated requirements, by changing the computing resources of the CPU, RAM and buffer capacity of the media device. With this design, the network's stability increases to cyber-attacks, as in the case of an attack on a virtualized network device, only a virtual router that handles the masked under the flow of attack will be overloaded. The novelty of this model is the structural-functional model introduces an automated block of the resource management manager that functionally responds to the hypervisor and, depending on the virtualization technology, uses part of the total resources of the CPU. This block in the conditions of traffic fluctuations dynamically allocates the computing resources of the network device for virtual routers, depending on the requirements of QoS flow. The computing resources of the device are understood as the hardware resources of the CPU, RAM, Buffer capacity, which configurations significantly affect the ability and performance of packet processing by the network node. To ensure the dynamic nature of the process of managing the computing resources within the proposed model virtualization of the network device can be by entering the control variables $\left\langle x_{i} y_{i} z_{i}\right\rangle$, responsible for the share of selected computing resources from the pool in the virtualization process of the i-th virtual router. Accordingly, ${ }^{x_{i}}$ for Buffer capacity, $y_{i}-\mathrm{CPU}, z_{i}$ - RAM, $n$ - number of

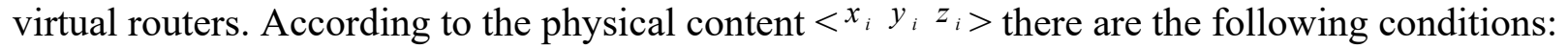

$$
\begin{gathered}
x_{i}, y_{i}, z_{i} \in\{0,1\}, \\
\sum_{i=1}^{n} x_{i}=1, \sum_{i=1}^{n} y_{i}=1, \sum_{i=1}^{n} z_{i}=1,
\end{gathered}
$$

The virtual queue length, processor's capacity, and virtual memory routine in a critical case cannot exceed the total buffer size, CPU, and RAM of the physical router without virtualization.

$$
\sum_{i=1}^{n} x_{i} \cdot q_{\text {черги }_{i}} \leq Q_{\text {буф }}, \quad \sum_{i=1}^{n} y_{i} \cdot C P U_{i} \leq C P U, \quad \sum_{i=1}^{n} z_{i} \cdot R A M_{i} \leq R A M,
$$


Where $q_{\text {queue }_{i}}$ - virtual queues packet length; $Q_{b u f}$ - volume of buffer`s memory; $C P U$ nominal processor`s frequency of physical router; $C P U_{i}$ - processor`s frequency $i$-th virtual router; $R A M$ - the physical memory of the physical router; $R A M_{i}$ - the physical memory of the $i_{\text {-го virtual router. }}$

$$
\left\{\begin{array} { l } 
{ t _ { \text { current } _ { i } } \leq T _ { \operatorname { m a x } _ { i } } } \\
{ p _ { \text { current } _ { i } } \leq P _ { \operatorname { m a x } _ { i } } } \\
{ d t _ { \text { current } _ { i } } \leq d T _ { \operatorname { m a x } _ { i } } }
\end{array} \Rightarrow \left\{\begin{array}{l}
x_{i} \cdot R A M_{i} \in R A M \\
y_{i} \cdot C P U_{i} \in C P U \\
z_{i} \cdot q_{\text {queue }_{i}} \in Q_{\text {buf }}
\end{array}\right.\right.
$$

Where $t_{\text {current }_{i}}$ - current delay for flow buffering for $i$-th virtual router; $T_{\max _{i}}$ - allowable flow delay for $i$-th virtual buffer of router according to established recommendations ; $p_{\text {current }_{i}}-$ the probability of dropping packets in the buffer for $i$-th virtual router; $P_{\max _{i}}$ - allowable flow loss in $i$-th virtual router's buffer according to established recommendations; $d t_{\text {current }_{i}}$, - current jitter flow buffering in $i$-th virtual router; $d T_{\max _{i}}$ - allowable flow's jitter in $i$-th virtual router's buffer according to established recommendations.

The paper proposes a method for decomposing the structure of a network router based on the theory of systems and mass-service networks, which made it possible to reduce fluctuations of the time parameters of service of various types of traffic that arise due to fluctuations of their intensity with the provision of QoS. A networked virtualization device according to the theory of queue can be represented by cascading buffer memory, servicing devices and resource manager (RMhypervisor) (fig. 2a). The loss of performance on the maintenance of the hypervisor is low, but it is necessary to take into account the impact of virtualization on the productivity of the system, depending on the virtualization technologies used in the process of designing corporate networks. Accordingly, in contrast to the known methods, improvements have been made in the work, namely, it is proposed to use the coefficient of virtualization impact on system performance $-\kappa$. The behaviour of multiservice IP network traffic is characterized by different distribution laws and therefore, when virtualization of a network device, each virtual router works with its class of services, each of which is described by the appropriate function of distributing intervals between packets and the function of distribution of service life. After decomposing the router virtualization model (fig. 2b) for a single type of traffic, we obtain a simplified single-server model.

An arbitrary mass service system can be used to describe such a model. For an example, we use the system $\mathrm{M} / \mathrm{M} / 1 / \mathrm{n}$, where calls to the input from the Poisson distribution law come into the input, in which a multiservice aggregate stream is formed. Consequently, for the proposed $\mathrm{M} / \mathrm{M} / 1 / \mathrm{n}$ system, the probability of loss in the $i-\mathrm{m}$ virtual router is determined by:

$$
P_{g m_{i}}=\frac{\left(1-\frac{\lambda_{i}}{\mu_{i} \cdot \kappa}\right) \cdot{\frac{\lambda_{i}}{\mu_{i} \cdot \kappa}}^{n_{i}}}{1-\frac{\lambda}{i}^{n+1} \cdot \kappa},
$$

Where $\lambda_{i}$ - the intensity of receiving of the packets $i$-th flow of a $i$-th virtual router; $\mu_{i-}$ the intensity of the packe processing $i$-th flow of a $i$-th virtual router; $n_{i}$ - the number of buffer spaces in a $i$-th virtual router. 


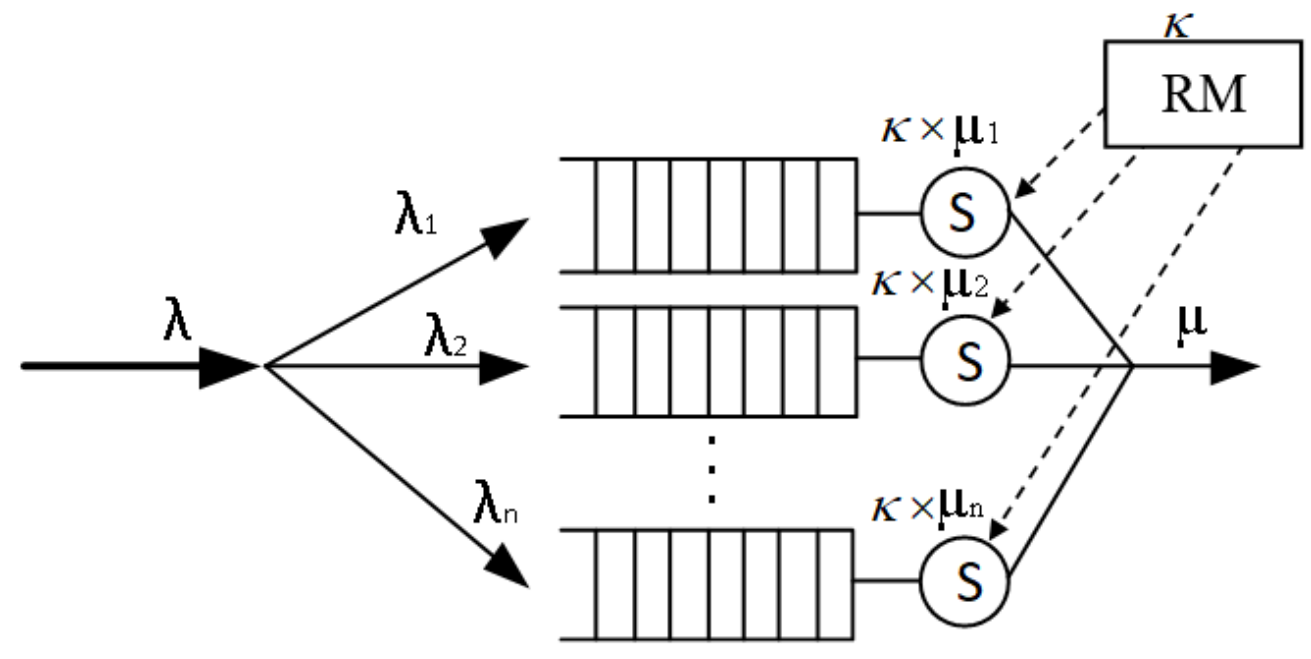

a)

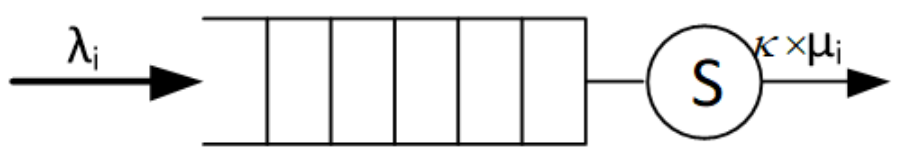

b)

Figure 2: Model of network device with virtualization (a) and virtual router model for IoT slice (b)

The average number of packages in the buffer of a $i$-th virtual router:

$$
\bar{N}_{i}=\frac{\frac{\lambda_{i}}{\mu_{i} \cdot \kappa}}{1-\frac{\lambda_{i}}{\mu_{i} \cdot \kappa}}-\frac{\left(1+n_{i}\right) \cdot{\frac{\lambda_{i}}{\mu_{i} \cdot \kappa_{i}}}^{n_{i}+1}}{1-{\frac{\lambda_{i}}{\mu_{i} \cdot \kappa}}^{n_{i}+1}},
$$

Accordingly, the average packet processing time in the $i$-th virtual router can be determined on the basis of Little's formula:

$$
\begin{gathered}
\bar{T}_{i}=\frac{\bar{N}_{i}}{\lambda_{i}} . \\
\bar{T}_{i}=\left(\frac{\frac{\lambda_{i}}{\mu_{i} \cdot \kappa}}{1-\frac{\lambda_{i}}{\mu_{i} \cdot \kappa}}-\frac{\left(1+n_{i}\right) \cdot \frac{\lambda_{i}}{\mu_{i} \cdot \kappa}}{1-\frac{\lambda_{i} n_{i}+1}{\mu_{i} \cdot \kappa}}\right) \cdot \frac{\kappa}{\lambda_{i}}
\end{gathered}
$$

For the first time, it is suggested to use the Noross formula for estimating the number of packets in the $i$-th buffer $i$-th virtual router, where each stream is characterized by its own properties and its own parameter of Hurst:

$$
N_{i}=\frac{\lambda_{i}}{\mu_{i} \cdot \kappa} \cdot \frac{\left(\frac{\lambda_{i}}{\mu_{i} \cdot \kappa}\right)^{\frac{H_{i}-0.5}{1-H_{i}}}}{\left(1-\frac{\lambda_{i}}{\mu_{i} \cdot \kappa}\right)^{\frac{H_{i}}{1-H_{i}}}},
$$


де $H_{i}$ - parameter of Hurst $i$-th flow, which is coming on $i$-th virtual router.

The virtual router buffer memory required is determined by the formula:

$$
Q_{i}=N_{i} \cdot L_{s r} \text {, }
$$

where $L_{s r}$ - average packet's length.

\section{Results and Discussion}

Thus, the task of resource planning is to choose the parameters of the structural and functional model of the service node, which ensure compliance with the necessary service quality parameters for information functionally oriented corporate networks. Services using virtual trunk routers allow the client to set up control over the private trunk network and ensure its security at a very low cost, as well as to organize virtual monoservice networks from the multiservice network (fig. 3). This figure shows how applications dedicated for each service can be virtualized and installed in each slice. With such an organization of multiservice networks, the problem of determining the model of incoming traffic is simplified, which, without decomposition, used complex properties of selfsimilarity. As seen so far, dedicated slices are created for services with different requirements. And virtualized network functions are being placed in physical device in each slice depending on services. Also, some network functions, like charging, policy control, etc., can be essential in one slice, but unnecessary in other slices. Operators can customize network slices the way they want, probably in the most cost-effective way.

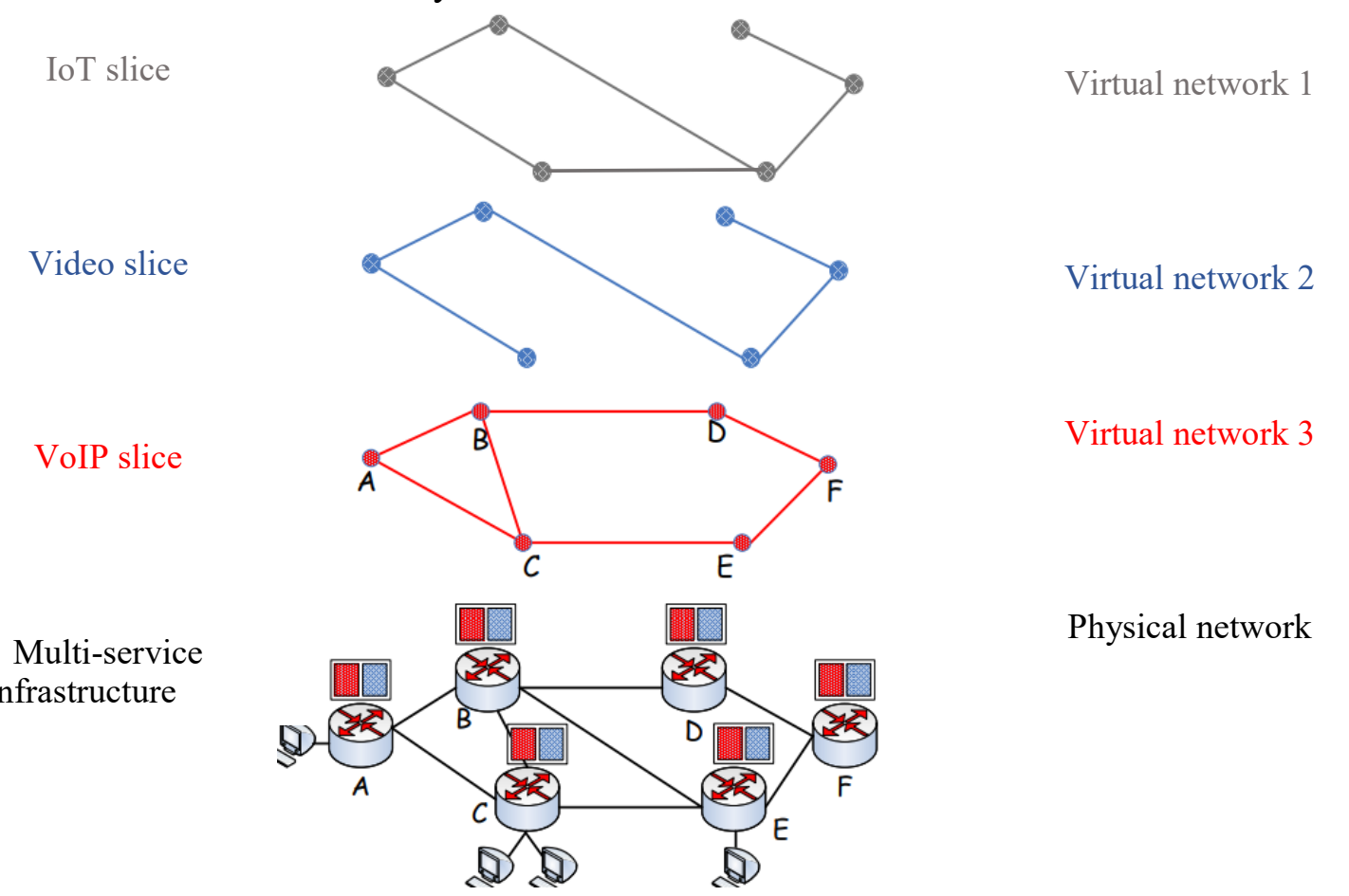

Figure 3: Network virtualization with decomposition on monoservice networks (network slicing)

The paper proposes an improved Norros's formula for determining the average number of packages of a particular service in the buffer of a virtual router. The formula for determining the packet delay duration of the $i$-th virtual network device is proposed throughout the source-todestination (7) and formula for determining the number of virtual nodes to provide QoS (8). 


$$
\begin{aligned}
& t_{i}=\sum_{k=1}^{M} \tau_{i k}+\sum_{j=1}^{N} \frac{\lambda_{i}}{\mu_{i} \cdot \kappa} \cdot \frac{\left(\frac{\lambda_{i}}{\mu_{i} \cdot \kappa}\right)^{\frac{H_{i}-0.5}{l-H_{i}}}}{\left(1-\frac{\lambda_{i}}{\mu_{i} \cdot \kappa}\right)^{\frac{H_{i}}{1-H_{i}}}} \cdot \frac{L_{s s_{i}} \cdot 8}{C_{j}}, \\
& N_{\text {node }_{i}}=\frac{T_{\max _{i} \cdot}}{\frac{\lambda_{i}}{\mu_{i} \cdot \kappa} \cdot \frac{\left(\frac{\lambda_{i}}{\mu_{i} \cdot \kappa}\right)^{\frac{H_{i}-0.5}{1-H_{i}}}}{\left(1-\frac{\lambda_{i}}{\mu_{i} \cdot \kappa}\right)^{\frac{H_{i}}{1-H_{i}}}} \cdot \frac{L_{s r_{i}} \cdot 8}{C_{j}}},
\end{aligned}
$$

where:

$M$ - the total number of communication channels between the two subscribers of the service;

$\tau_{i k}$ - the amount of delay time of the distribution of the service of the $i$-th priority service on the k-channel of communication $i$ - th virtual router;

$N$ - the total number of networks devices with virtualization of resources located between two subscribers of the service; $C_{j}$ - bandwidth for $j$-th channel.

Investigation of the duration of the delay of the $i$-th virtual router over the entire source-todestination transmission path was carried out on the basis of the network model shown in Fig. 3 using the proposed formula 7 and the effectiveness of its use compared to the Norros's formula, which does not use the average number of packages. The length of the delay in distributing the service packet of $i$-th priority over the k-channel is taken for everyone the same $\tau_{i k}=1 \mathrm{~ms}$, based on the fact that the random process of the delay time is the duration of packet buffering in the nodes, which is difficult to predict due to the influence of many factors, especially with complex queuing algorithms with priorities. As we see from the fig. 4 model is constructed so that when passing the packet from the source to the addressee, take into account different instances of the loading factors of the virtual nodes where queues with different virtual zones of boost resource loading arise.

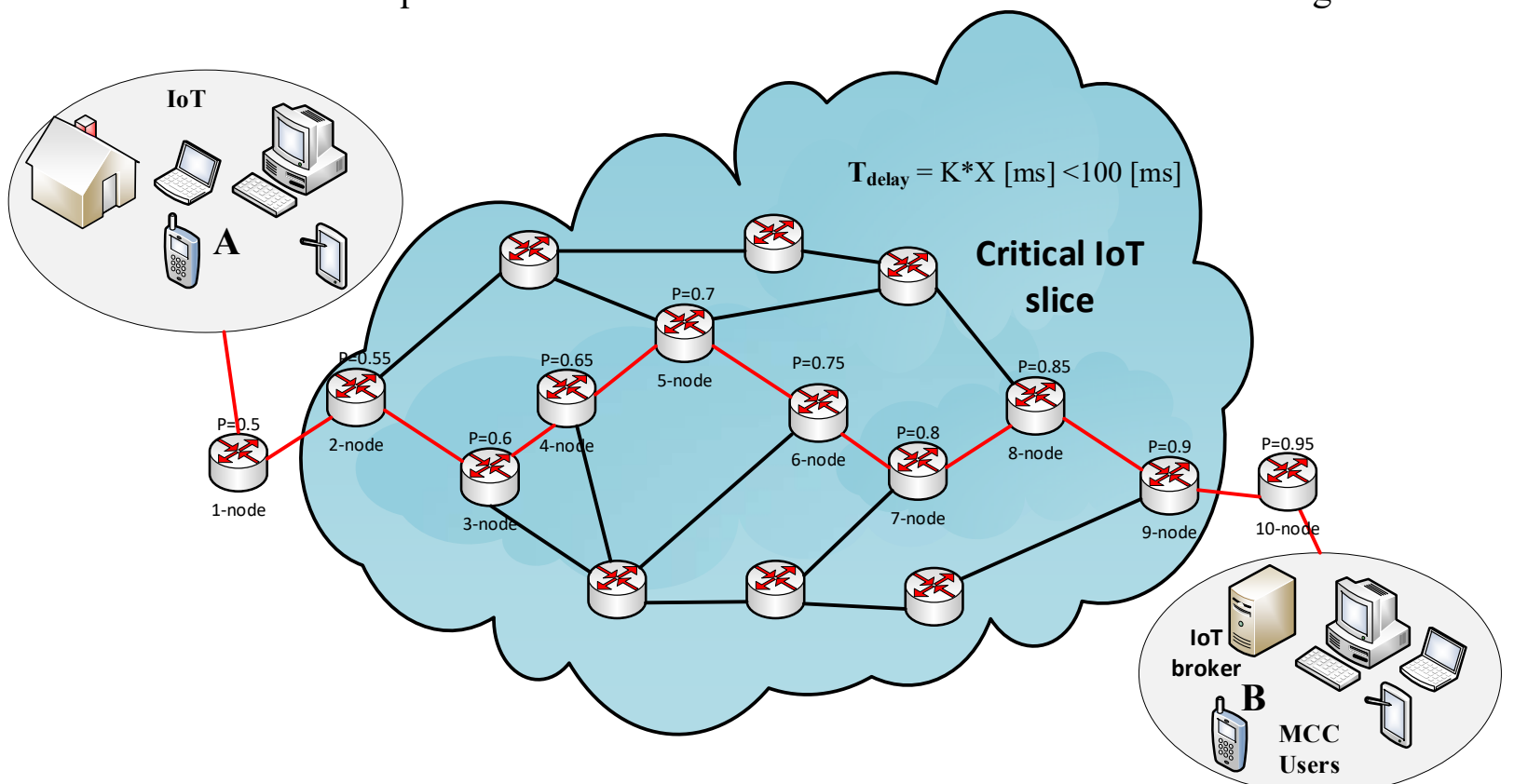

Figure 4: Model of the investigated virtualized network for predicting the delay time 
Prediction was made for traffic generated by groups of users who use different services in different volumes. The graphs for forecasting delay of IoT services times for the $\mathrm{i}$-th virtual router are shown in fig. 5 .

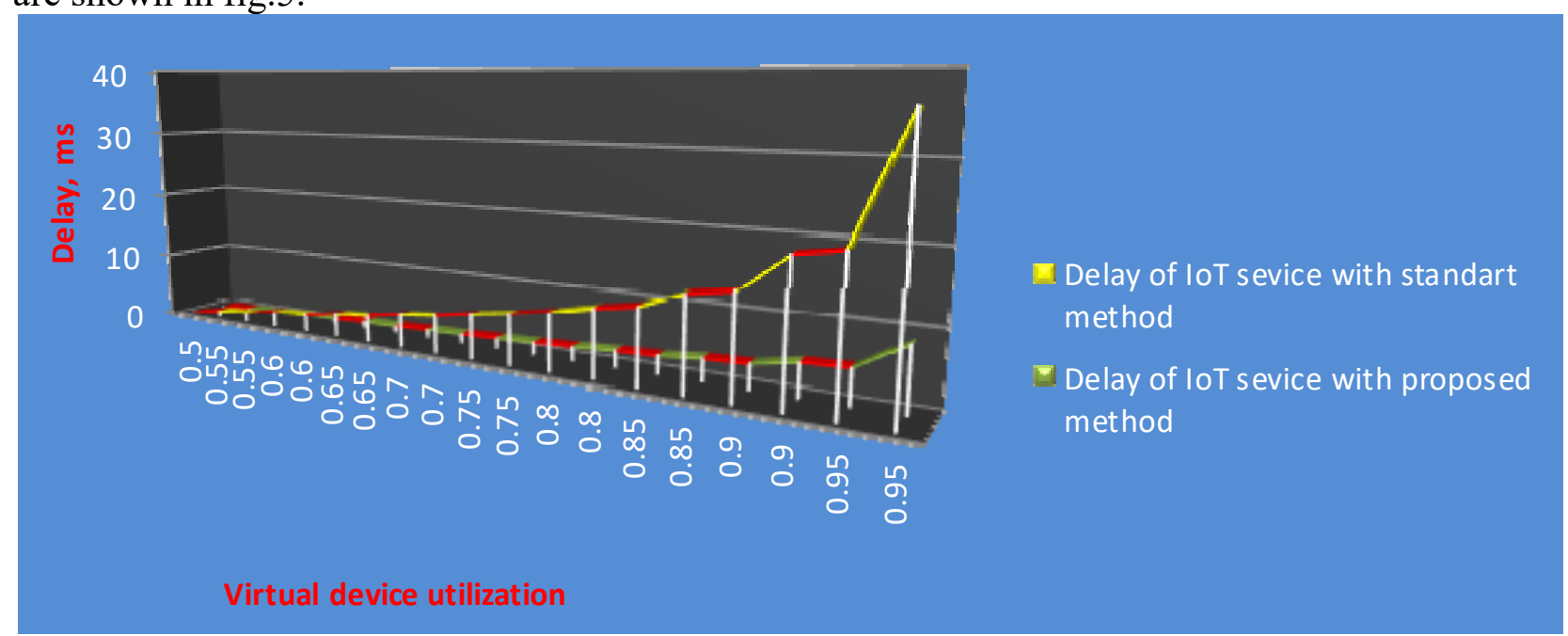

Figure 5: Forecasting the delay duration of IoT services packets without and with virtualization resourses

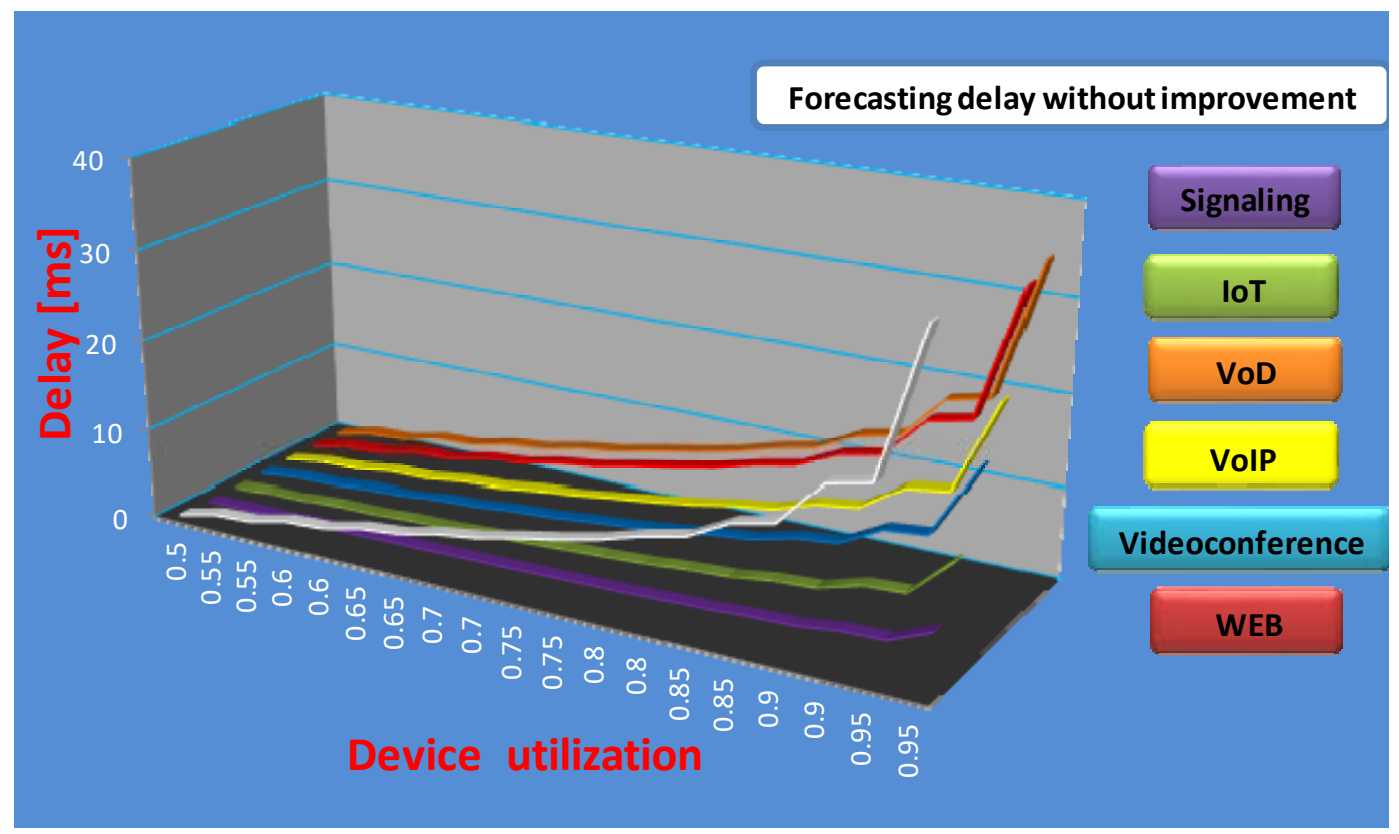

Figure 6: Prediction of the service packet delay for the i-th virtual router of the researched network

Figure 6 shows the effectiveness of the proposed formula (11), which determines the duration of the packet delay of the $\mathrm{i}$-th virtual router over the entire transmission path from source to destination. Compared to the results of forecasting the latency of packets defined by the formula when we do not know the number of packages and services that are in the buffer. Namely according to the formula known - $\mathrm{ti}=36.1 \mathrm{~ms}$, and the formula improved $-\mathrm{ti}=9.8 \mathrm{~ms}$. As we can see, the effectiveness of forecasting the duration of IoT packet delay has increased 3.6 times the traffic generated by a group of home users. From Fig. 6 determines the effectiveness of predicting the latency of packets for all services transmitted on the network by a group of home users, as shown in 
Table 1, where $\mathrm{k}$ is the coefficient of increasing the efficiency of predicting the duration of service packet delays.

Table 1. The effectiveness of predicting the latency of service packages of the proposed formula

\begin{tabular}{|c|c|}
\hline Service & K \\
\hline IoT & 3,656941813 \\
\hline Videoconferencing & 2,056688548 \\
\hline VoIP & 1,589633977 \\
\hline Internet data & 1,093147158 \\
\hline Media on request & 1,0520732 \\
\hline Interactive data & 1,022597916 \\
\hline
\end{tabular}

From Fig. 7 is defined, the prediction of the number of virtual nodes on the network, which provides QoS at different values of the average download factor for users. And is showed the efficiency of prediction from the application of the improved formula (12) for real-time services and critical IoT services.

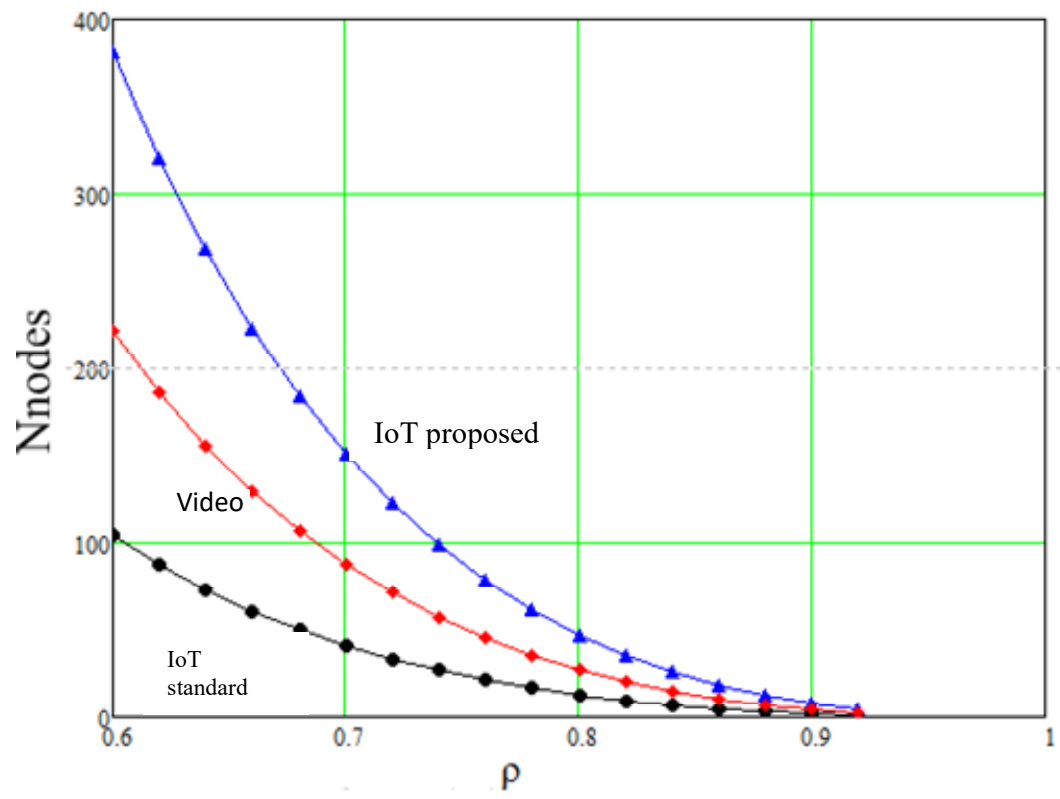

Figure 7: Maximum number of network nodes which assure the demanded QoS for real-time services and critical IoT services

The packet delays in network node and the maximum number of nodes to assure the demanded service quality have been forecasted and calculated.

\section{Conclusions}

The mathematical model of a network device with virtual routers was proposed. Based on this mathematical model the method of decomposing the structure of the node was developed, which enables to determine the basic parameters of the system of virtual queues based on the FIFO algorithm for the purpose of analysis the efficiency using of network resources, as well as the definition of the quality parameters of servicing the traffic flows of the services provided for a given the intensity of the arrival of packets on the input interface of the network element. The approach to predicting the duration of service packets delay on the i-th virtual router was offered based on 
which it is possible to obtain results with higher reliability, total packets buffering time of a certain service when passing through virtual nodes with different loading rates at the design stage for various functional-oriented corporate networks. Also, the maximum number of transit nodes that can be installed for the quality provision of multiservice services was calculated. The estimated number of transit nodes for IoT was 3.6 times higher than the number of transit logistics data after virtualization.

\section{Acknowledgements}

This research is supported by the project entitled "Methods for designing the heterogeneous information and communication system to deploy the software defined multi-purpose 5G networks", funded by Ukrainian government and by the project No. 0117 U007177 "Designing the methods of adaptive radio resource management in LTE-U mobile networks for $4 \mathrm{G} / 5 \mathrm{G}$ development in Ukraine," funded by Ukrainian government.

\section{References}

[1] A. Azzarà and L. Mottola, "Virtual resources for the Internet of Things," 2015 IEEE 2nd World Forum on Internet of Things (WF-IoT), Milan, 2015, pp. 245-250.

[2] B. Fekade, T. Maksymyuk, M. Kyryk and M. Jo, "Probabilistic Recovery of Incomplete Sensed Data in IoT," in IEEE Internet of Things Journal.

[3] K. Fujikawa, V. P. Kafle, P. Martinez-Julia, A. H. A. Muktadir and H. Harai, "Automatic Construction of NameBound Virtual Networks for IoT," 2017 IEEE 41st Annual Computer Software and Applications Conference (COMPSAC), Turin, 2017, pp. 529-537.

[4] V. Eramo, A. Cianfrani, E. Miucci, M. Listanti, D. Carletti and L. Gentilini, "Virtualization and virtual router migration: Application and experimental validation," 2014 26th International Teletraffic Congress (ITC), Karlskrona, 2014, pp. 1-6.

[5] M. Beshley, M. Seliuchenko, O. Panchenko, O. Zyuzko and I. Kahalo, "Experimental performance analysis of software-defined network switch and controller," 2018 14th International Conference on Advanced Trends in Radioelecrtronics, Telecommunications and Computer Engineering (TCSET), Lviv-Slavske, Ukraine, 2018, pp. 282286.

[6] V. Romanchuk, M. Beshley, O. Panchenko and P. Arthur, "Design of software router with a modular structure and automatic deployment at virtual nodes," 2017 2nd International Conference on Advanced Information and Communication Technologies (AICT), Lviv, 2017, pp. 295-298.

[7] Beshley M. SOA quality management subsystem on the basis of load balancing method using fuzzy sets / M. Beshley, M. Klymash, B.Strykhalyuk, O. Shpur, B. Bugil, I. Kagalo // International Journal of Computer Science and Software Engineering (IJCSSE). - 2015 - Volume 4 - Issue 1 - P.10-21

[8] H. Beshley, M. Beshley, T. Maksymyuk and I. Strykhalyuk, "Method of centralized resource allocation in virtualized small cells network with IoT overlay," 2018 14th International Conference on Advanced Trends in Radioelecrtronics, Telecommunications and Computer Engineering (TCSET), Lviv-Slavske, Ukraine, 2018, pp. 1147-1151.

[9] M. Klymash, H. Beshley, M. Seliuchenko and M. Beshley, "Algorithm for clusterization, aggregation and prioritization of M2M devices in heterogeneous 4G/5G network," 2017 4th International Scientific-Practical Conference Problems of Infocommunications. Science and Technology (PIC S\&T), Kharkov, 2017, pp. 182-186.

[10] M. Klymash, H. Beshley, O. Panchenko and M. Beshley, "Method for optimal use of 4G/5G heterogeneous network resourses under M2M/IoT traffic growth conditions," 2017 International Conference on Information and Telecommunication Technologies and Radio Electronics (UkrMiCo), Odessa, 2017, pp. 1-5.

[11] O. Panchenko, A. Polishuk, M. Seliuchenko and M. Beshley, "Method for adaptive client oriented management of quality of service in integrated SDN/CLOUD networks," 2017 4th International Scientific-Practical Conference Problems of Infocommunications. Science and Technology (PIC S\&T), Kharkov, 2017, pp. 452-455

[12] M. Klymash, V. Romanchuk, M. Beshley and P. Arthur, "Investigation and simulation of system for data flow processing in multiservice nodes using virtualization mechanisms," 2017 IEEE First Ukraine Conference on Electrical and Computer Engineering (UKRCON), Kiev, 2017, pp. 989-992. 
[13] M. Klymash, M. Beshley and B. Stryhaluk, "System for increasing quality of service of multimedia data in convergent networks," 2014 First International Scientific-Practical Conference Problems of Infocommunications Science and Technology, Kharkov, 2014, pp. 63-66.

[14]M. Beshley, V. Romanchuk, V. Chervenets and A. Masiuk, "Ensuring the quality of service flows in multiservice infrastructure based on network node virtualization," 2016 International Conference Radio Electronics \& Info Communications (UkrMiCo), Kiev, 2016, pp. 1-3..

[15] M. Klymash, M. Beshley, B. Strykhalyuk and T. Maksymyuk, "Research and development the methods of quality of service provision in Mobile Cloud systems," 2014 IEEE International Black Sea Conference on Communications and Networking (BlackSeaCom), Odessa, 2014, pp. 160-164

[16] M. Seliuchenko, M. Beshley, O. Panchenko and M. Klymash, "Development of monitoring system for end-to-end packet delay measurement in software-defined networks, " IEEE International Conference on Modern Problems of Radio Engineering, Telecommunications and Computer Science (TCSET'2016), Feb. 23-26, 2016, Lviv, Ukraine, pp. $667-670$.

[17] V. Romanchuk, M. Beshley, A. Polishuk and M. Seliuchenko, "Method for processing multiservice traffic in network node based on adaptive management of buffer resource," 2018 14th International Conference on Advanced Trends in Radioelecrtronics, Telecommunications and Computer Engineering (TCSET), Lviv-Slavske, Ukraine, 2018, pp. 1118-1122.

[18] H. Esaki and R. Nakamura, "Overlaying and slicing for IoT era based on internet's end-to-end discipline," 2017 IEEE International Symposium on Local and Metropolitan Area Networks (LANMAN), Osaka, 2017, pp. 1-6. 